\title{
PREDICTIVE MODELS FOR TRANSIENT LOADS OF THE VERTICAL STABILIZER OF AN AIRCRAFT DEVELOPED USING CANONICAN CORRELATION ANALISION
}

\author{
Michał Dziendzikowski \\ Wojciech Zieliński \\ Lukasz Obrycki \\ Marta Woch \\ Piotr Synaszko \\ Krzysztof Dragan \\ Andrzej Leski
}

Air Force Institute of Technology, ul. Ks. Boleslawa 6, 01-494 Warsaw, Poland

\begin{abstract}
Knowledge about loads occurring in the structure during aircraft operation is vital from the point of view of the damage tolerance approach to aircraft design. In the best-life scenario, such information could be available from a network of sensors, e.g. strain gauges, installed in the aircraft structure to measure local stresses. However, operational loads monitoring (OLM) systems are still not widely applied. Instead, what is available is a set of flight parameters, which by the laws of inertia and aerodynamics help determine the dominant part of loads acting on a given element. This paper discusses the canonical correlation analysis (CCA) as a method for selecting the flight parameters used to predict aircraft loads. CCA allows for the identification of both different modes of stress distribution as well as flight parameters which are best suited for their prediction. The paper presents the application of this method to identify loads acting on the vertical stabilizer of an aircraft.
\end{abstract}

Keywords: damage tolerance, loads prediction, loads monitoring.

\section{INTRODUCTION}

It's been over 40 years since the damage tolerance philosophy was introduced into the aircraft design process [1]. Of vital importance from the point of view of maintaining structural integrity of an aircraft is the ability to control the assumed spectrum of loads in the structure. Loads distribution under the assumed profile of aircraft operation is a central point in the aircraft design process (Fig. 1). Loads in the structure determine intervals between subsequent non-destructive inspections (NDI) providing information about the condition of the aircraft structure. Because the way a particular aircraft is operated after its introduction into service does not necessarily fit its pre-assumed profile, the scheduled NDI timeline may be no longer appropriate. This used to contribute to flight accidents, such as the 1988 accident of Boeing 737 of the Aloha Airlines, which was subjected to the excessive number of cabin pressurizing cycles, accelerating the development of WFD (Widespread Fatigue Damage) [2]. 


\section{THE METHODOLOGY OF THE APPROACH}

As mentioned, there is a need to monitor the actual loads spectrum of particular aircraft. In the modern approach, Operational Loads Monitoring (OLM) systems are designed to perform this task (Figure 2). Such systems are based on networks of sensors, e.g. strain gauges, Fiber Bragg Gratings - FBG (optical sensors), which allow for assessment of loads in given elements of the aircraft structure [3]. What is available for older constructions, or those for which OLM system installation is not economically efficient, is a set of flight parameters, which by the laws of inertia and aerodynamics should determine dominant part of loads, acting on every element of the aircraft structure. Thus, the stress at a given instant of time $t$ for a fixed point $\mathbf{r}$ of a given element of the structure is a function of fight parameters $p_{1}(t), \ldots, p_{N}(t)$ :

$$
\hat{\sigma}(\mathbf{r}, t)=f\left(p_{1}(t), \ldots, p_{N}(t)\right)
$$

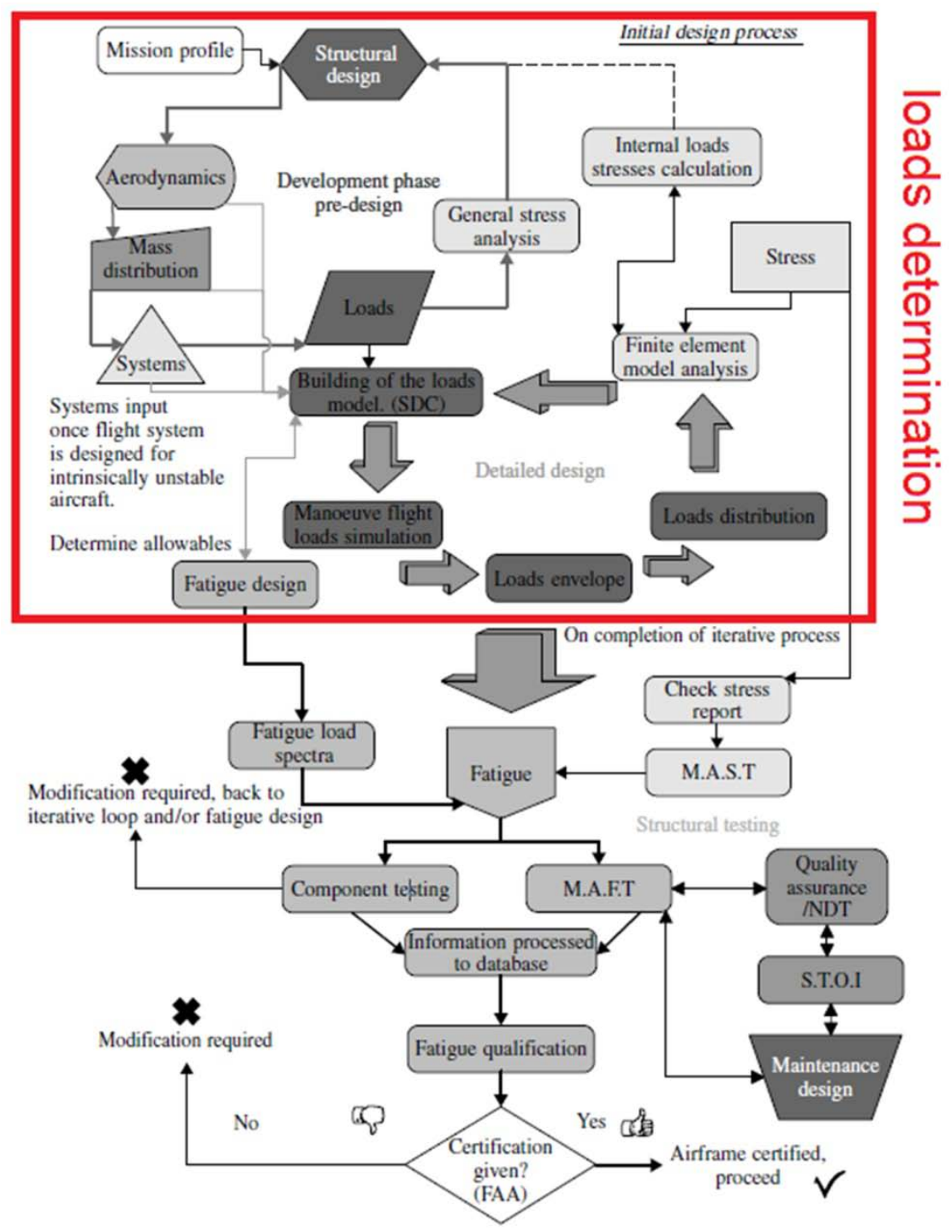

Figure 1. Initial aircraft design process [4] 


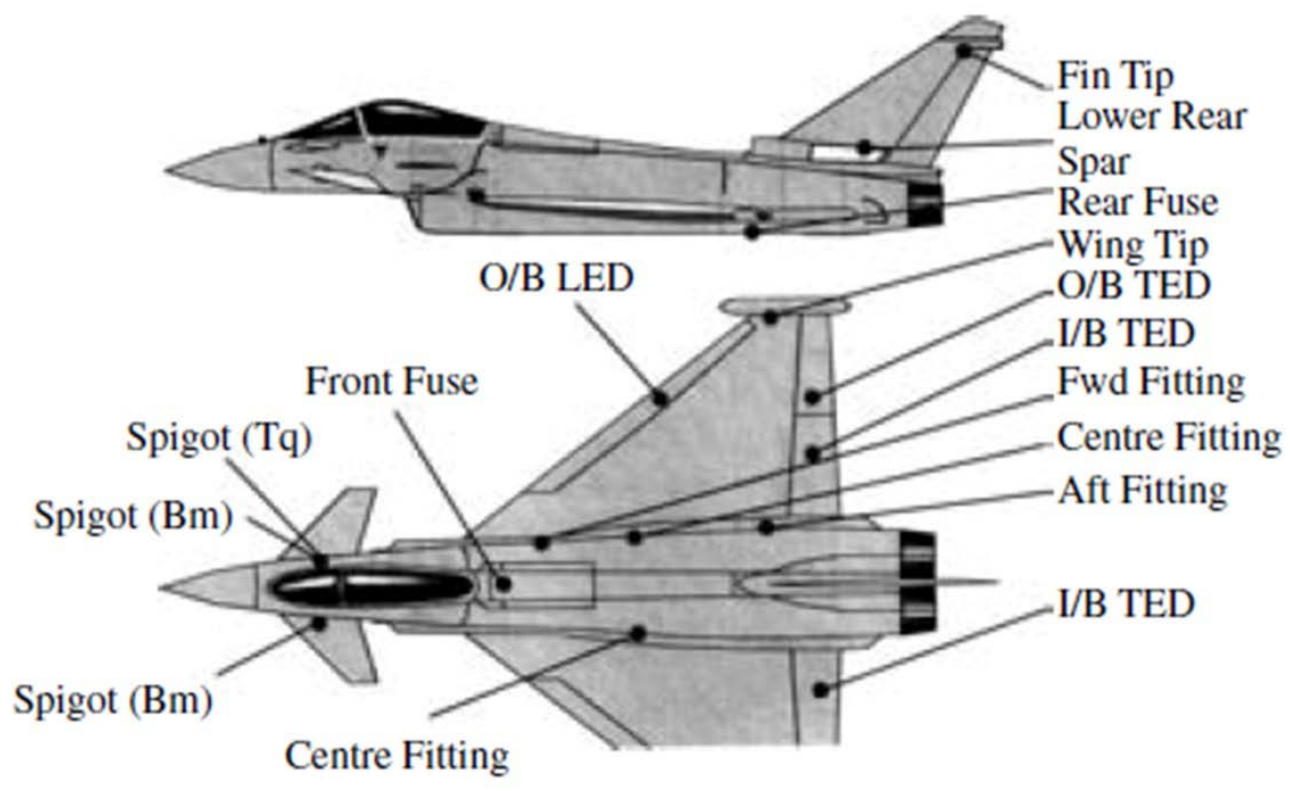

Figure 2. Location of sensors used for the health and usage monitoring of Eurofighter aircraft [5]

There are several issues related to loads monitoring via their functional dependence on flight parameters. Firstly, to properly assess the fatigue of an element, it is necessary to distinct different stress distributions, for which fatigue cycles should be counted. Sensors, e.g. strain gauges used for OLM purposes, respond simultaneously to a given type of loads. Thus, there is a need to determine how many distinct states of load (Fig. 3) are encountered during aircraft operation, and how often they occur, based on signals registered from the installed network of sensors. In addition, if monitoring is to be based on flight parameters, for each state of load one should select a subset of flight parameters that determine this state and the functional form between this subset and a given stress distribution.
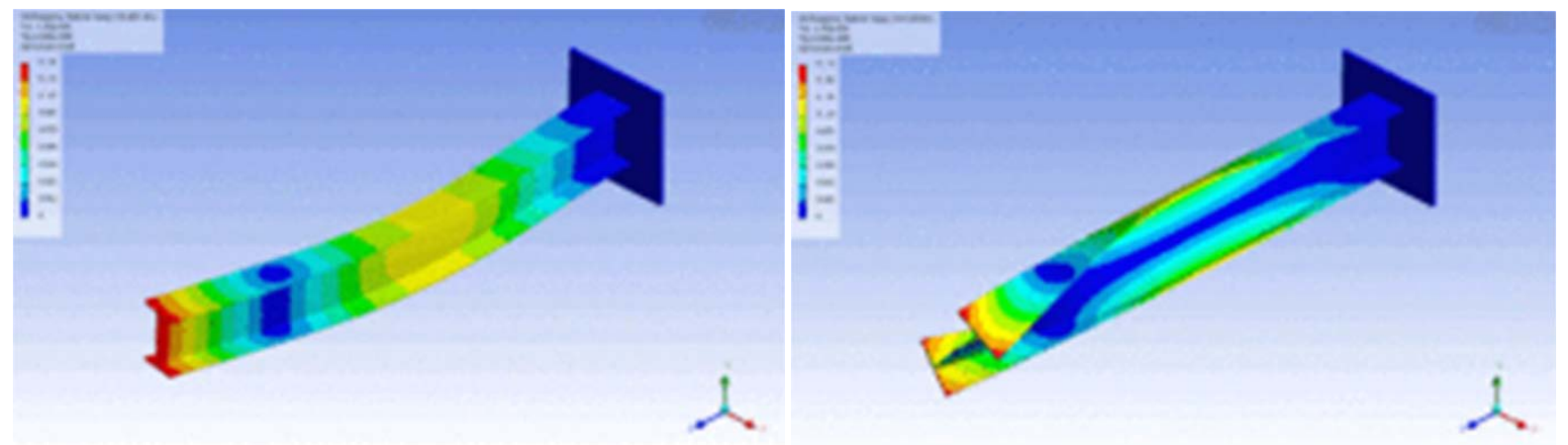

Figure 3. Different modes of a beam deflection [6]

For the two issues, i.e. determination of loads states and set of flight parameters best suited for their prediction, Canonical Correlation Analysis (CCA) [7] can be used. Given two sets of variables, i.e. response variables, e.g. measured stresses $\left\{\sigma_{1}, \ldots, \sigma_{M}\right\}\left\{\sigma_{1}, \ldots, \sigma_{M}\right\}$ and predictor variables, e.g. flight parameters $\left\{p_{1}, \ldots, p_{N}\right\}$, one has to find the uncorrelated linear combinations of $\left\{\sigma_{1}, \ldots, \sigma_{M}\right\},\left\{p_{1}, \ldots, p_{N}\right\}$, such that the correlation between them is subsequently maximized, i.e. $\sigma_{1}^{c}$, $p_{1}^{c}$. The first two canonical combinations $\sigma_{1}^{c}, p_{1}^{c}$ satisfy: 


$$
\begin{aligned}
& \sigma_{1}^{c}=\sum_{j=1}^{N} \alpha_{1}^{j} \sigma_{j}, \quad p_{1}^{c}=\sum_{j=1}^{M} a_{1}^{j} p_{j}, \\
& \operatorname{cor}\left(\sigma_{1}^{c}, p_{1}^{c}\right)=\max _{\tilde{\alpha}, \tilde{a}} \operatorname{cor}\left(\sum_{j=1}^{N} \tilde{\alpha}^{j} \sigma_{j}, \sum_{j=1}^{M} \tilde{a}^{j} p_{j}\right),
\end{aligned}
$$

whereas the next two combinations need to be uncorrelated with the first pair:

$$
\begin{aligned}
& \sigma_{2}^{c}=\sum_{j=1}^{N} \alpha_{2}^{j} \sigma_{j}, \quad p_{1}^{c}=\sum_{j=1}^{M} a_{2}^{j} p_{j}, \\
& \operatorname{cor}\left(\sigma_{2}^{c}, p_{2}^{c}\right)=\max _{\tilde{\alpha}, \tilde{a}} \operatorname{cor}\left(\sum_{j=1}^{N} \tilde{\alpha}^{j} \sigma_{j}, \sum_{j=1}^{M} \tilde{a}^{j} p_{j}\right), \quad \text { and } \operatorname{cor}\left(\sigma_{1}^{c}, \sigma_{2}^{c}\right)=\operatorname{cor}\left(p_{1}^{c}, p_{2}^{c}\right)=0
\end{aligned}
$$

and analogously for every subsequent pair of canonical combinations. Thus, CCA is an equally suitable method of determining different stress distributions as the Principal Component Analysis (PCA) is [7]. However, it has the advantage of allowing for the preliminary selection of the flight parameters appropriate for prediction of different states of load of the structure.

\section{DETERMINING LOADS OF THE VERTICAL STABILIZER OF THE MIG-29}

The CCA method was used to determine the stress distribution in MiG-29's vertical stabilizer. The aircraft's structure is presented in Figure 4.

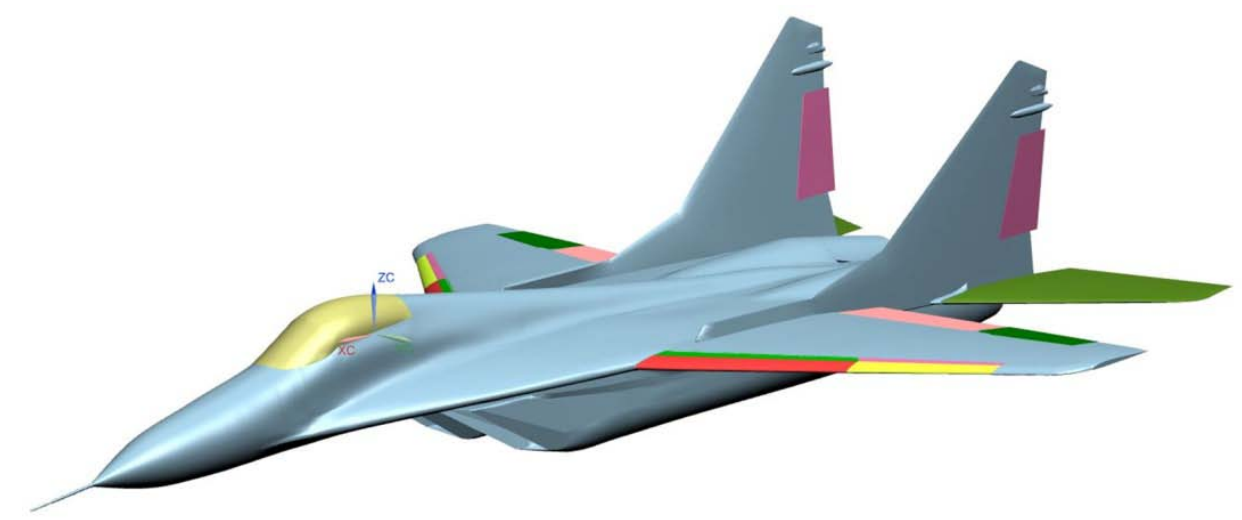

Figure 4. A scheme of MiG-29 aircraft

The stress distribution is the sum of two components: $\hat{\sigma}_{\text {inert }}$ coming from inertial forces and $\hat{\sigma}_{\text {aero }}$ resulting from aerodynamic forces:

$$
\hat{\sigma}(\mathbf{r}, t)=\hat{\sigma}_{\text {inert }}(\mathbf{r}, t)+\hat{\sigma}_{\text {aero }}(\mathbf{r}, t) .
$$

For the vertical stabilizer, the second component was considered as the main contributor to stress distribution. The following flight parameters were initially considered for the modeling of $\hat{\sigma}_{\text {aero }}$ :

- $V$ - the velocity of aircraft relative to the atmosphere;

- $\quad H$ - the altitude of aircraft;

- $\alpha$ - the attack angle;

- $\quad \beta$ - the sideslip angle;

- $\quad \dot{\gamma}$ - the roll speed;

- $\delta$ - the rudder inclination. 
The function relating the flight parameters and stress was assumed to be of the following form:

$$
\sigma=\frac{\rho_{0} V_{r z}^{2}}{2}\left(1-\frac{H}{44300}\right)^{4.256}\left(\tilde{\sigma}_{\text {nozzle }}\left(V_{r z}, \alpha\right)+\tilde{\sigma}_{\text {slip }}\left(\beta, V_{r z}, \alpha\right)+\tilde{\sigma}_{\text {roll }}\left(\dot{\gamma}, V_{r z}, \alpha\right)+\tilde{\sigma}_{\text {rudder }}\left(\delta, V_{r z}, \alpha\right)\right) \text {, }
$$

where $\rho_{0}$ is the air density at the ground level according to the International Standard Atmosphere (ISA) model, $\tilde{\sigma}_{\text {nozzle }}, \tilde{\sigma}_{\text {slip }}, \tilde{\sigma}_{\text {roll }}, \tilde{\sigma}_{\text {rudder }}$ are dimensionless functions describing the contribution to stress distribution due to:

- the Venturi effect, i.e. MiG-29 aircraft has two vertical stabilizers (Fig. 4) which can be considered as a nozzle;

- the sideslip angle;

- the rate of rotation;

- the position of the rudder.

Thus the first factor in the Eq. 5 describes the aerodynamic pressure at the altitude $H$, and functions $\tilde{\sigma}_{\text {nozzle }}, \tilde{\sigma}_{\text {slip }}, \tilde{\sigma}_{\text {roll }}, \tilde{\sigma}_{\text {rudder }}$ should give the stress distribution for different maneuvers of the aircraft. As the exact form of $\tilde{\sigma}_{\text {nozzle }}, \tilde{\sigma}_{\text {slip }}, \tilde{\sigma}_{\text {roll }}, \tilde{\sigma}_{\text {rudder }}$ functions was not known, for CCA application the second order Taylor expansion was used, e.g:

$$
\tilde{\sigma}_{\text {nozzle }}=c_{V} V+c_{V V} V^{2}+c_{\alpha} \alpha+c_{\alpha \alpha} \alpha^{2}+c_{V \alpha} V \alpha \text {, }
$$

with unknown constants $c_{V}, c_{V V}, c_{\alpha}, c_{\alpha \alpha}, c_{V \alpha}$, etc. All the variables resulting from that expansion, e.g. $V, V^{2}, \alpha, \alpha^{2}$, were considered as independent input variables to the CCA model, i.e. a new set of flight parameters was considered.

The CCA model was applied based on the data obtained during the flight test campaign of two MiG-29 aircraft. Thus, in total, there were 4 independent installations of the OLM systems:

- OLM1 - mounted on the left stabilizer of the first aircraft;

- OLM2 - mounted on the right stabilizer of the first aircraft;

- OLM3 - mounted on the left stabilizer of the second aircraft;

- OLM4 - mounted on the right stabilizer of the second aircraft.

The CCA model was trained using only the data obtained by the OLM1 installation and tested on the rest strain gauges corresponding to OLM2, OLM3 and OLM4.

A single mode of stress distribution was noticed as a result of CCA. The first canonical flight parameter $p_{1}^{c}$ was in good correspondence with the data obtained for about $70 \%$ of strain gauges of OLM2, OLM3, OLM4 - those showing the highest amplitude of the records. The remaining strain gauges exhibited small variations during flights and were too noised to be useful. Examples of the correspondence between the normalized canonical parameter and normalized records of a strain gauge obtained during steady and high maneuver flights are presented in Figure 5. The leading contribution to the canonical parameter was due to $\tilde{\sigma}_{\text {nozzle }}$ contribution. 

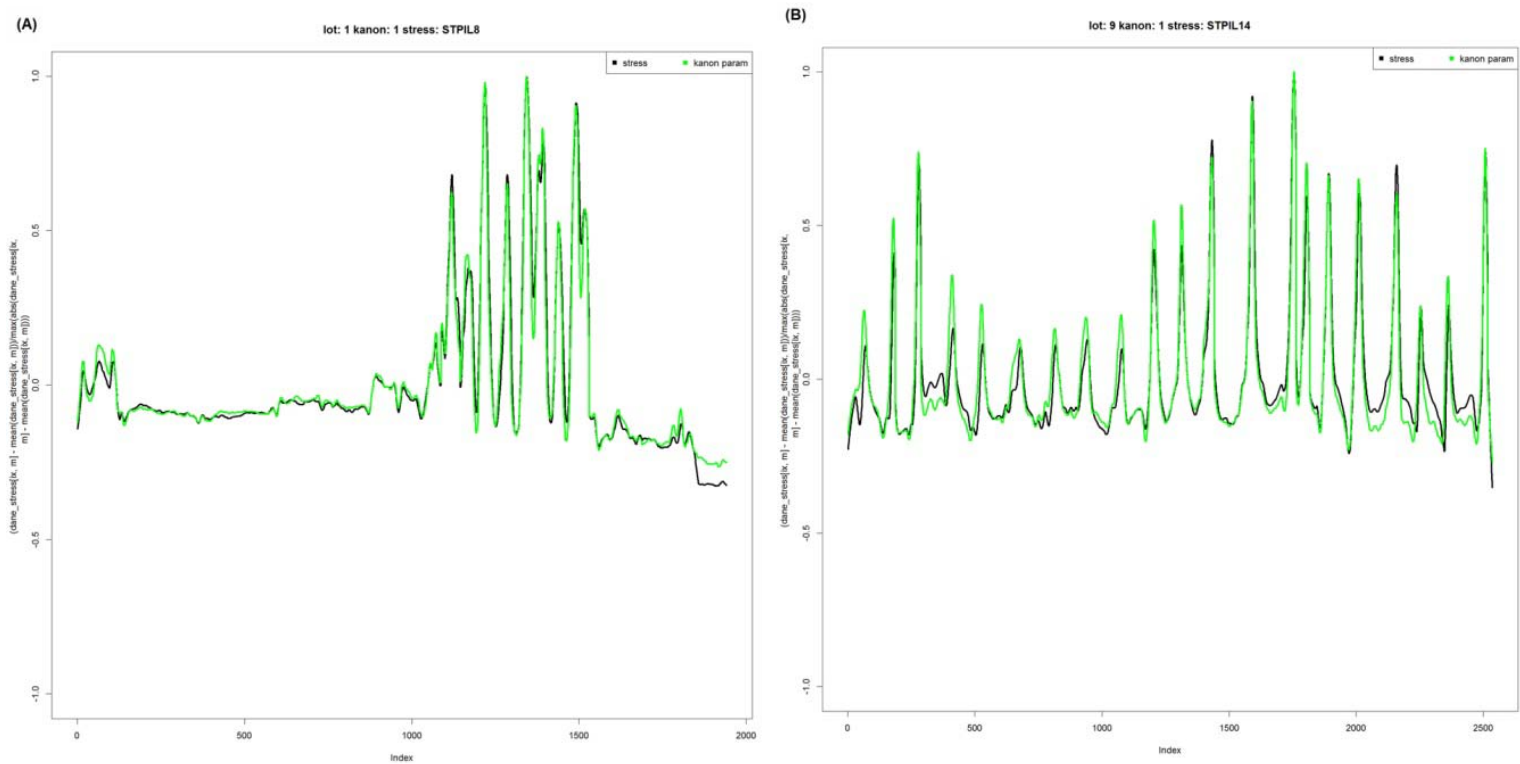

Figure 5. Examples of record of strain gauge (black) and canonical flight parameter (green) for: (a) steady and (b) high maneuver flights

\section{SUMMARY}

The paper presented Canonical Correlation Analysis (CCA) as a method of selecting flight parameters well suited to predict loads of the aircraft structure. CCA allows both for the identification of different modes of stress distribution as well as for the identification of flight parameters which are the best suited for their prediction. The preliminary results of the application of this method in order to identify loads acting on the vertical stabilizer of MiG-29 aircraft were also presented.

\section{REFERENCES}

[1] C. Osgood. Fatigue Design - 2nd edition. Pergamon Press, 1982.

[2] United States National Transportation Safety Board. Aircraft Accident Report NTSB/AAR-89/03.

[3] C. Boller and W.J Staszewski. Aircraft structural health and usage monitoring. In W.J. Staszewski, C. Boller, and Tomlinson G.R., editors, Health Monitoring of Aerospace Structures, pages 29-73. John Wiley and Sons, Ltd, 2004.

[4] Banks, D. 2000. A Look Into the World of Structural Health Monitoring, Industrial Placement Report, EADS/Sheffield University.

[5] Boller, C. 2001. Ways and options for aircraft structural health management, Smart Materials and Structures, Vol. 10, pp. 432-439.

[6] https://en.wikipedia.org/wiki/Bending

[7] T. Hastie, R. Tibshirani, J. Friedman: The Elements of Staistical Learning: Data Mining, Inference, and Prediction, second ed., Springer Science+Business Media, New York, 2009. 